\title{
Directed Assembly of Single Wall Carbon Nanotube
}

\section{Field Effect Transistors}

Erika Penzo ${ }^{1 \dagger}$, Matteo Palma ${ }^{1 \dagger \dagger}$, Daniel A. Chenet ${ }^{2}$, Geyou Ao ${ }^{3}$, Ming Zheng ${ }^{3}$, James C. Hone ${ }^{2}$, and Shalom J. Wind ${ }^{1 *}$

${ }^{1}$ Department of Applied Physics and Applied Mathematics, Columbia University, New York, NY, USA $;{ }^{2}$ Department of Mechanical Engineering, Columbia University, New York, NY, USA; ${ }^{3}$ National Institute of Standards and Technology, Gaithersburg, MD, USA.

†Present address: The Molecular Foundry, Lawrence Berkeley National Laboratory, Berkeley, CA; ${ }^{\dagger}$ Present address: Department of Chemistry \& Biochemistry, School of Biological and Chemical Sciences, Queen Mary University of London, London, UK. 

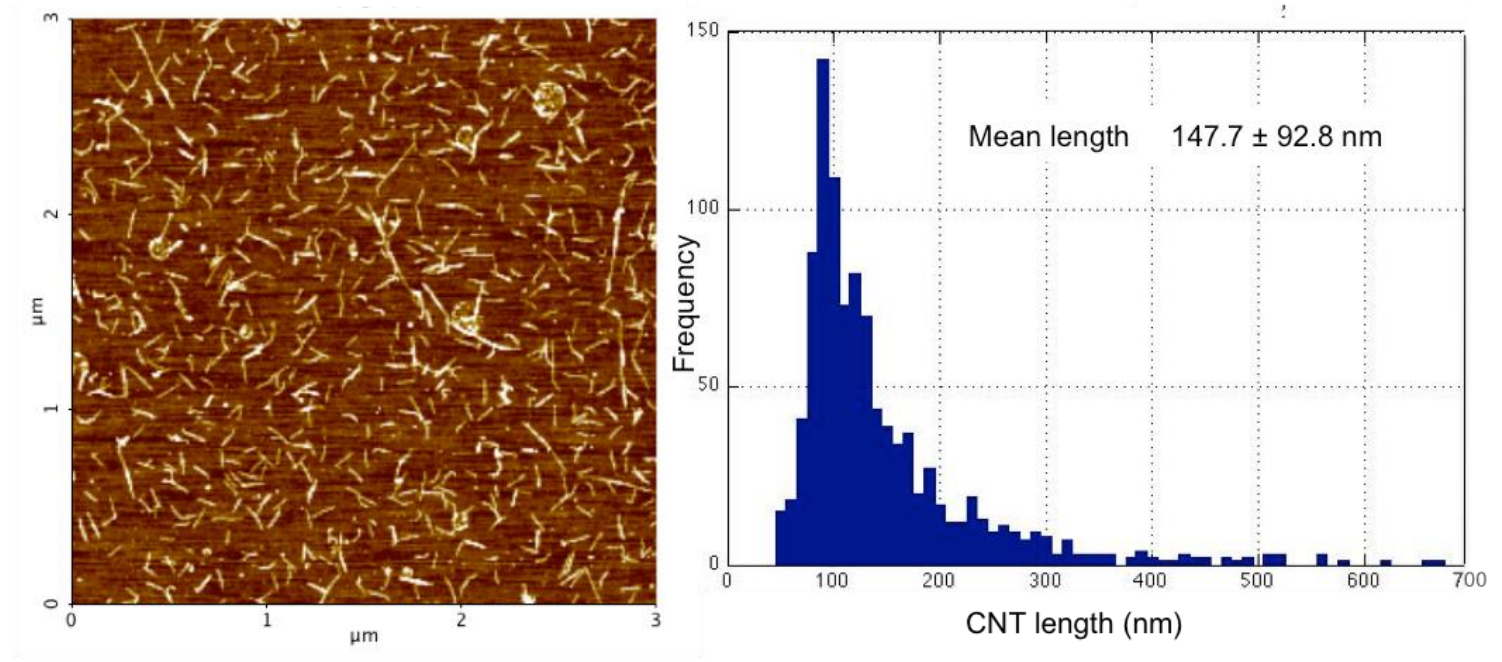

Figure S1. AFM image of DNA-wrapped SWCNT segments (mixed chirality solution) deposited on $\mathrm{SiO}_{2}$ substrates (left). Histogram of the DNA-wrapped SWCNT segments size distribution (right). The average length of $(147.7 \pm 92.8) \mathrm{nm}$ is determined from $c a$. 1,000 objects. 


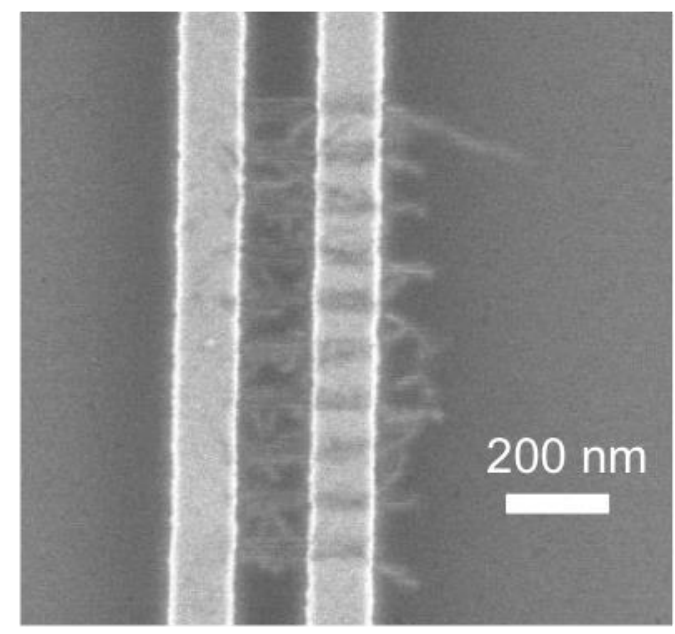

Figure S2. SEM images of DNA-wrapped SWCNT segments assembled from solution (mixed chirality solution) onto substrates with pre-patterned $\mathrm{Au}$ electrodes. The $\mathrm{SiO}_{2}$ surface and the $\mathrm{Au}$ electrodes were passivated with a PEG layer. The PEG was selectively removed by e-beam patterning of PMMA and reactive ion etching. The patterned line width was $30 \mathrm{~nm}$. Multiple SWCNT segments adsorbed on each line, and they oriented at various angles. The lateral shift in the position of the nanotube segments with respect to the electrodes is due to registration inaccuracy in the e-beam patterning. 


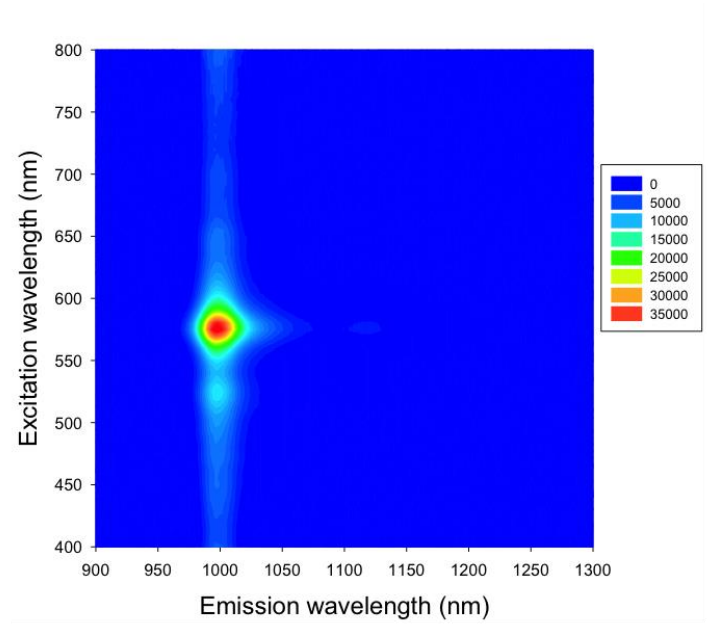

Figure S3. NIR fluorescence map acquired using a spectrofluorometer equipped with a liquid nitrogen cooled InGaAs detector. The nanotube sample was diluted to an OD of about 0.5 for the measurement. The spectrum was corrected for lamp excitation, filter transmission, and detector efficiency. 


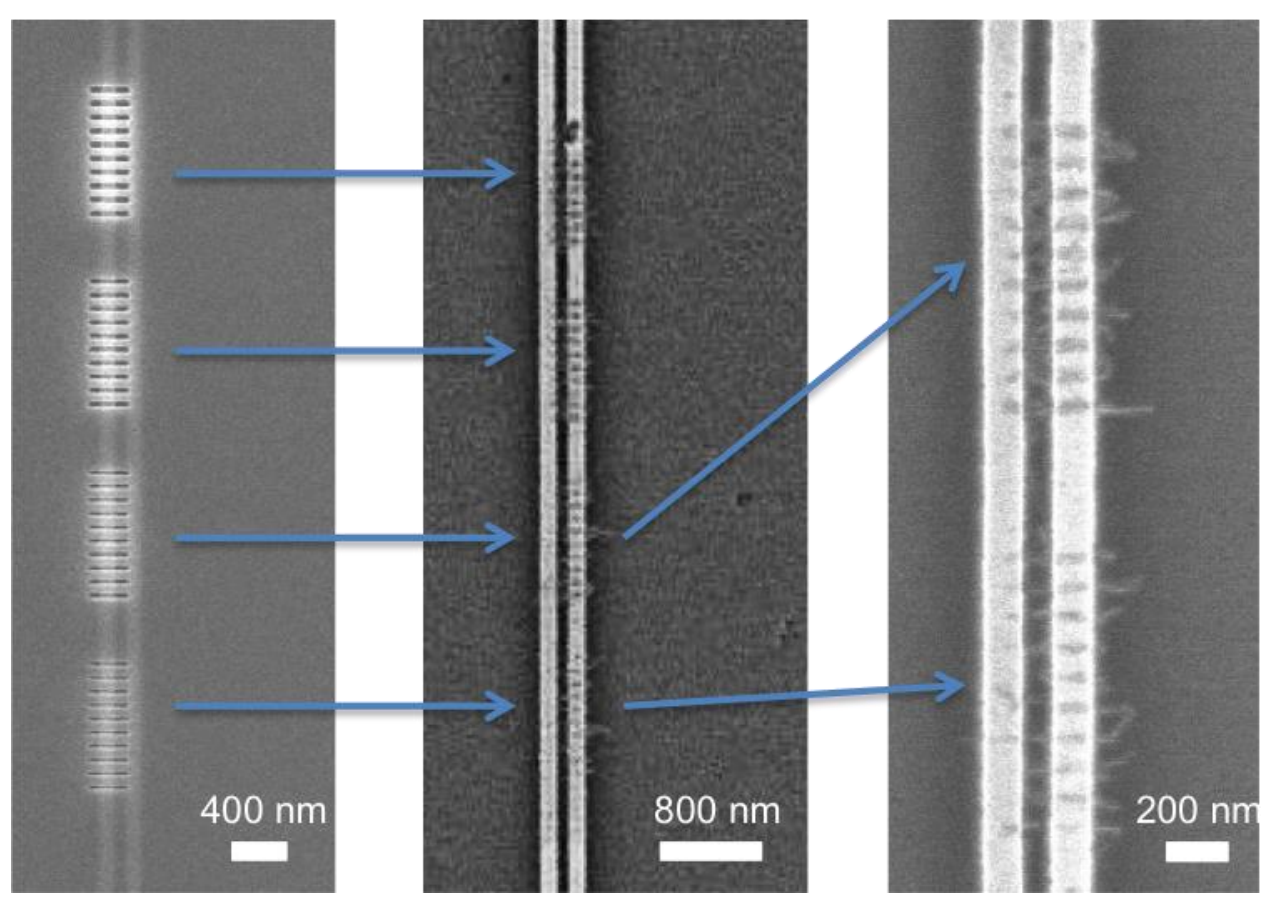

Figure S4. SEM images of (left) a Ti mask with arrays of patterned lines with different thickness - from the top $40 \mathrm{~nm}, 30 \mathrm{~nm}, 20 \mathrm{~nm}$, and $10 \mathrm{~nm}$ - and of the corresponding assembled SWCNT segments (center and right). The images are from three different samples patterned with the same pattern. The different horizontal offset of the lines array respect to the electrodes is due to registration inaccuracy in the e-beam patterning. 


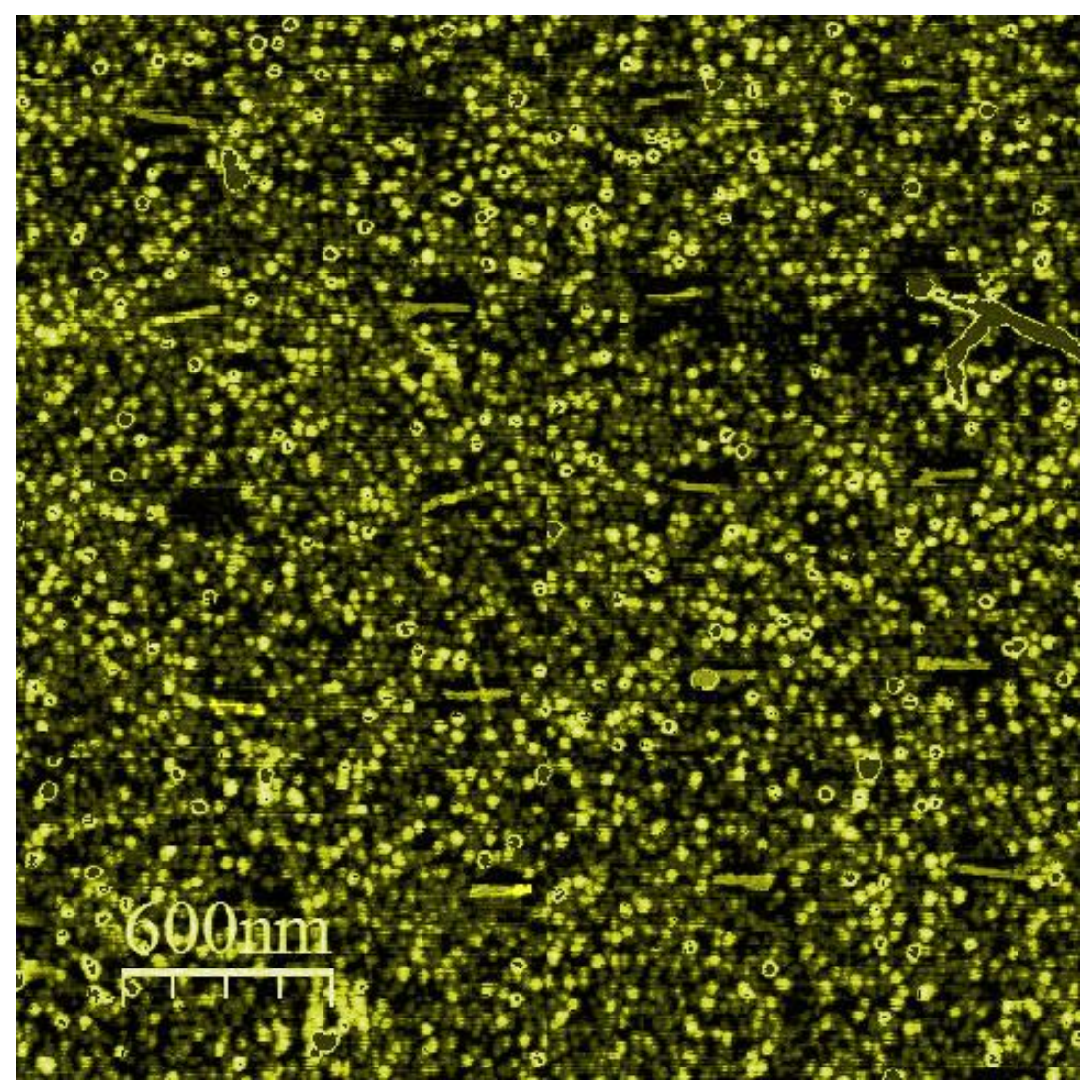

Figure S5. AFM image of an array of SWCNTs (mixed chirality) deposited on a PEG passivated substrate, where the PEG had been removed selectively form rectangular regions spaced $500 \mathrm{~nm}$ apart. 

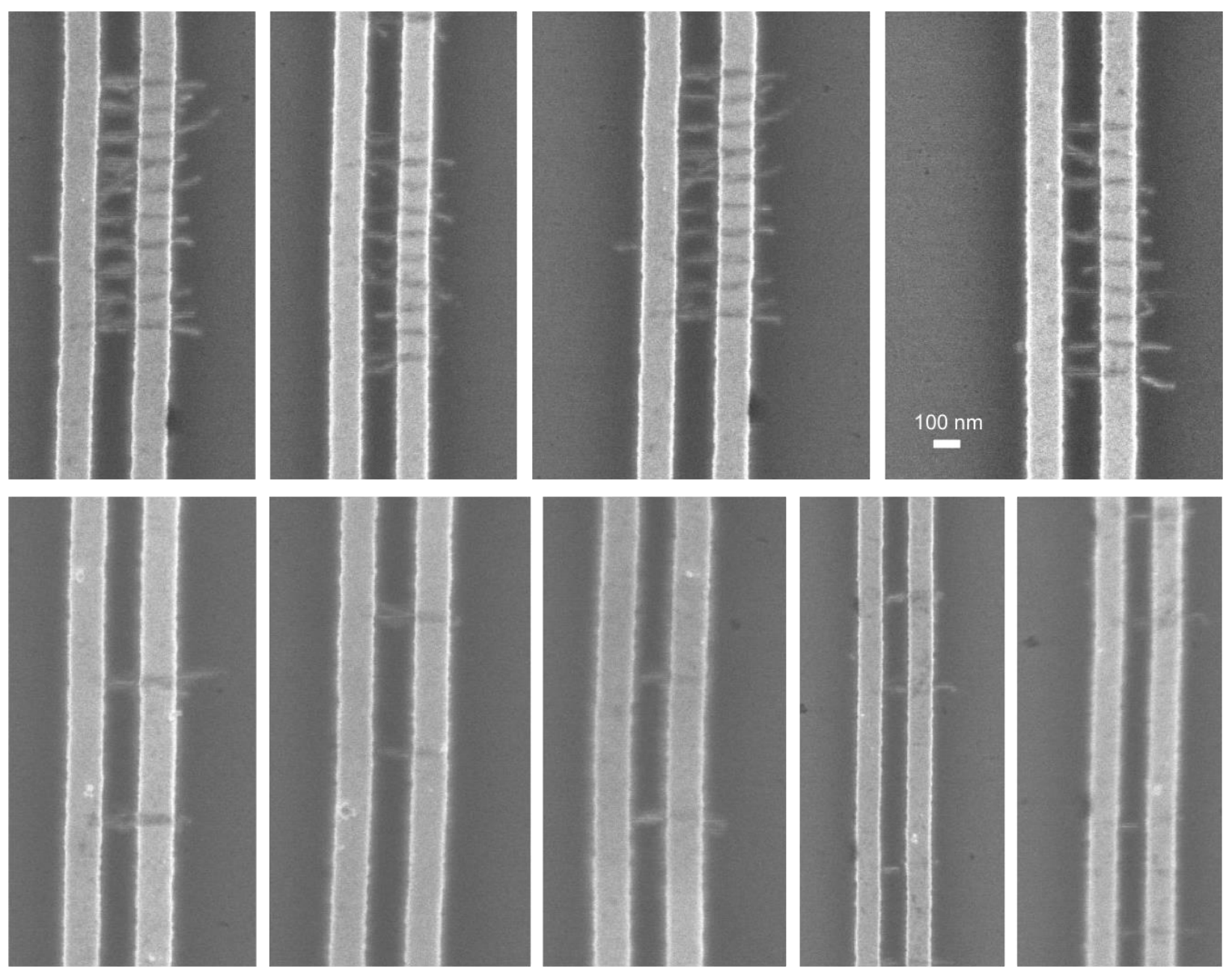

Figure S6. SEM images of arrays of SWCNTs deposited on prepatterned hydrophilic lines arranged in (top) ten lines spaced $100 \mathrm{~nm}$ apart with each array separated by $500 \mathrm{~nm}$ (see Figure S4) and in (bottom) two lines spaced $500 \mathrm{~nm}$ apart with each array separated by $1 \mu \mathrm{m}$. The dense arrays show a small number of tubes crossing multiple lines while the sparse arrays show individual nanotube placement without any misplaced tube. 